\title{
Experiences by patients and health professionals of a multidisciplinary intervention for long-term orofacial pain
}

\author{
Håkan Nilsson' \\ Mats Samuelsson ${ }^{2}$ \\ Susanne Ekdahl ${ }^{3}$ \\ Yvonne Halling ${ }^{4}$ \\ Anders Öster ${ }^{4}$ \\ Kent-Inge Perseius ${ }^{2,3}$ \\ 'Department of Stomatognathic \\ Physiology, Faculty of Odontology, \\ Malmö University, and Unit of \\ Stomatognathic Physiology, Specialist \\ Dental Centre, Kalmar County \\ Hospital, Kalmar, ${ }^{2}$ Department \\ of Neurobiology, Caring Sciences \\ and Society, Karolinska Institutet, \\ Stockholm, ${ }^{3}$ Nyckeln Competence \\ Center for Pedagogics in Healthcare, \\ Kalmar County Hospital, Kalmar, \\ ${ }^{4}$ Unit of Stomatognathic Physiology, \\ Specialist Dental Centre, Kalmar \\ County Hospital, Kalmar, Sweden
}

Correspondence: Kent-Inge Perseius Nyckeln Competence Center for Pedagogics in Healthcare, Kalmar County Hospital, S-39I 85 Kalmar, Sweden Email kentingep@Itkalmar.se
This article was published in the following Dove Press journal:

Journal of Multidisciplinary Healthcare

20 September 2013

Number of times this article has been viewed

\begin{abstract}
The aim of the present study was to describe patients' and health professionals' experiences of a multidisciplinary stress-focused clinical evaluation with prolonged engagement as an intervention for patients with long-term orofacial pain. Data in the patient part of this study were collected by free-text questionnaires using open-ended questions. Data were collected by group interview in the part of the study concerning health professionals. All data were analyzed according to qualitative content analysis. Data from patients revealed three categories for the intervention, ie, "helpful for most and crucial for some", "being listened to, respected and validated", and "gives important coping strategies". The results showed that a vast majority of patients described themselves as having been helped by the intervention. Some patients reported that meeting with the orofacial pain consultant team was crucial to the future course of their lives. Most patients described still having residual pain and symptoms, and only a few described their pain as being fully remitted. However, because of the intervention, the patients reported being able to adopt more constructive coping strategies. They also described their perception of the pain as being different, in that it was not so frightening once they had been given a model with which to understand it. Data from the health professionals revealed similar categories. Concordance between the patients' and health professionals' experiences was striking. In their descriptions, the health professionals and patients underscored the same components as being effective, with understanding, respect, and validation being the most important. The multidisciplinary approach was highlighted as being key to success by both the patients and health professionals.
\end{abstract}

Keywords: long-term orofacial pain, clinical evaluation, stress reduction, prolonged engagement, teamwork, qualitative research

\section{Introduction}

Long-term orofacial pain (duration $\geq 3$ months) is a severe health problem that is strongly associated with symptoms of anxiety and depression, as well as reduced work capacity and early retirement. ${ }^{1-3}$ Like other pain syndromes, orofacial pain seems to be a common health problem. Epidemiologic studies show that $21 \%-30 \%$ of the population suffers from orofacial pain. ${ }^{4,5}$ The prevalence is higher in women and in the younger age groups. ${ }^{4}$ The often long-term nature of the condition is reflected in the finding that more than half of those suffering from orofacial pain have not recovered on follow-up 4 years later. ${ }^{5}$

In recent years, there has been a growing body of scientific work highlighting different aspects of this problem. Studies have shown that long-term orofacial pain is comorbid with fibromyalgia, sleeping disorders, cardiovascular problems, and gas- 
trointestinal problems, suggesting that orofacial pain may be part of a more general stress-related health problem. ${ }^{6,7}$ In a clinical context, variation in the presentation of orofacial pain leads patients to different specialists, including general practitioners, dentists, neurologists, orthopedists, chiropractors, and psychiatrists, who provide a variety of treatments, often with little collaboration and recognition of the biopsychosocial background of the condition. ${ }^{8}$

Impairment of psychosocial functioning in patients with long-term pain has been shown to be strongly associated with pain-related cognitions and beliefs. ${ }^{9,10}$ Thus, there is emerging agreement in the scientific literature that behavioral and educational strategies are useful in the treatment and management of such pain. ${ }^{9,11}$ These strategies consist of teaching patients self-management and self-control over not only pain symptoms but also their cognitive attributions or meanings, which seems to help them to maintain a productive level of psychosocial function, even if the pain itself is not absent. ${ }^{9,11}$ Research on the use of behavioral and educational interventions for long-term low back pain, for instance, has been more common in recent years, but the data for interventions focusing on long-term orofacial pain are still scarce. Further, published data describing patients' and health professionals' experiences around such interventions seem to be nonexistent.

The aim of the present study was to describe patients' and health professionals' experiences of a multidisciplinary stress-focused clinical evaluation with prolonged engagement as an intervention for patients with long-term orofacial pain.

\section{Materials and methods Clinical evaluation}

The clinical evaluation in this study was conducted by a consultant team connected to a special unit for orofacial pain at a hospital-based specialist dental clinic in the southeast of Sweden. The team consists of six persons, ie, a general practitioner, a specialist physician in rehabilitation medicine, a dentist specialized in orofacial pain, a psychologist, a physical therapist, and a dental nurse specialized in stress therapy methods (referred to as a "stress therapist" below).

Patients referred to the unit for evaluation of chronic orofacial pain were all potential participants for further evaluation and prolonged engagement with the consultant team. The orofacial pain specialist and the stress therapist meet and clinically examine referred patients with a thorough anamnesis. Patients who have a long, complicated medical history of widespread pain with repeated medical visits and without proper pain reduction are referred to the consultant team.

The central component of the clinical evaluation is a 2-hour session in which the patient meets the entire consultant team at the same time. The session is divided into four phases.

The first phase is the patient's narrative, in which the patient gives her/his own picture of their pain and related symptoms and life situation, as well as earlier experiences of treatment. The team actively listens and validates the patient's experiences with small comments like "that must have been tough" and "how strong of you". The patient's narrative is also illuminated and made more specific through comments and open-ended questions from the team members, such as "tell us more" and "in what way?"

The second phase comprises a pedagogic explanation, where team members discuss the patient's narrative and provide medical and scientifically based explanations for the presenting symptoms and problems. This is done with help from stress vulnerability models, psychoneuroimmunology, and health psychology, ie, an integrative (psycho-neuroendocrine-immune) model drawn from pain-related and stress-related research. ${ }^{12,13}$ In this process, different pedagogic visualizing techniques are used, including whiteboard, PowerPoint presentations, and brochures.

The third phase involves relevance testing, during which the patient, in discussion with the team, tests the relevance of the explanatory models for her/his specific problems.

The fourth phase includes searching for helpful strategies and summarizing them. The team suggests self-care strategies as well as further treatment and support from dental or health caregivers to help with specific problems. Examples might include pain self-care classes, short-term focused psychotherapy or stress management lessons, and getting a bite splint or/and an appointment with a specialist pain physician to revise medication. This is then summarized into an "action plan". In some cases, the action plan is supported by referrals to other caregivers.

Prolonged engagement consists of a follow-up phase (up to 6 months), during which the stress therapist maintains regular contact with the patient to check on how the action plan is working. The therapist checks in once a week at the start but less frequently as time goes by.

Preparation for the team session consists of assessment by two of the team members, ie, the orofacial pain specialist and the stress therapist. This session has several purposes, ie, collecting complementary data by anamnesis, photodocumentation of pain localization, and screening for stress 
in the form of autonomous dysfunction using the Stress and Crisis Inventory (SCI 93). ${ }^{14}$ The patient also receives a written presentation and expert opinions from members of the consultant team. Last, the conference room where the team session is to take place is shown to the patient so that he/she can prepare mentally for the session. During the session, that patient has the opportunity to ask questions and to discuss points which may be difficult to understand. The patient at this time also gives written consent for the health care team to obtain earlier patient files and log notes from health and dental providers. Before the team session, all team members prepare by thoroughly reading all the material arising from the assessment session. On the team session day, the team meets an hour before the patient's arrival in order to discuss the material.

\section{Participants}

Patients were enrolled consecutively for the patient part of this study. All patients who had been referred to the orofacial pain consultant team during the study period $(\mathrm{n}=31,25$ women and six men) were asked to participate. Twentythree $(74 \%)$ of these patients, comprising 18 women and five men, gave their written consent and fulfilled the data collection criteria. A dropout analysis was done for the remaining eight individuals (see information below). For the health professional part of the study, all six members of the aforementioned orofacial pain consultant team (four women and two men) were asked to participate. All provided their verbal consent to participate and fulfilled their responsibilities for data collection.

\section{Data collection}

Data in the patient part of the study was collected by freetext questionnaires containing open-ended questions, which were administered by mail. The patients were allowed 3 weeks to complete and return the questionnaires, which were addressed to the premises of the authors SE and KIP (a county council center of expertise for pedagogy in health care). The questionnaire focused on two specific areas, ie, the patients' experiences of symptoms and suffering and their life situations before and after the intervention, as well as experiences of contact with the consultant team and their perceptions of whether they had been helped or not. The questionnaire was structured using a funnel approach that started with wide and open questions, including "Can you describe what your life situation was like before your meeting with the consultant team?" and "What is your life situation like today?" These were followed by more specific questions and examples. For the nonresponders, a reminder was send out 3 weeks after the original invitation, giving them a further 2 weeks to respond.

In the health professional part of the study, data were collected by a group interview that took place at the specialist dental clinic in the hospital and lasted 90 minutes. The interview guide focused on one question area, ie, the team members' experiences working with the intervention and developing an action plan. The interview guide was used freely, allowing the respondents to narrate experiences in their own words. Questions were asked using a funnel approach, starting with wide, open-ended questions like "Why did you start to work within this team?" followed by more specific questions and questions that asked for examples. The interview was audiotaped. The authors SE and KIP undertook all data collection.

\section{Data analysis}

The data in the free-text questionnaires as well as the group interview were analyzed by qualitative content analysis according to Burnard. ${ }^{15}$ Content analysis is a method concerned with meanings, intentions, consequences, and context. ${ }^{16}$ The aim of such an analysis is to produce a detailed and systematic recording of themes and issues addressed in text-based materials and to link the themes together under a reasonably exhaustive category system. ${ }^{1}$ The text from the questionnaires and the audiotaped group interview were transcribed verbatim into a word processing data program. Analysis of the material from the two study groups proceeded through the following stages: the material was read as openmindedly as possible in order to gain an overall impression of "what this is about"; the text was reread several times; units of meaning were identified by open coding; the meaning units were condensed; the condensed meaning units were abstracted and organized into a preliminary system of categories in interplay with the text as a whole; the authors SE and KIP independently analyzed the data in steps 2, 3, and 4, to enhance trustworthiness; and the authors HN, SE and KIP reflected on and discussed the codes and preliminary category system until they reached agreement. In all, they agreed on 74 meaning units and nine categories.

\section{Dropout analysis}

The eight patients who did not respond to either the original invitation letter or the reminder were contacted by telephone (by author KIP) to find out whether their experiences differed in any major way from those of the responders. One of them could not be reached, one had developed dementia, 
and one did not want to talk at all. These three individuals were excluded from the study. The remaining five individuals stated that they had been too busy and/or too tired to participate in the study and would not like to participate in an audiotaped telephone interview. They were then asked if they could answer one short question without being recorded, ie, whether they were satisfied or dissatisfied with their meeting with the orofacial team (which was considered the major source of selection bias). Four of them stated that they were satisfied, and one reported dissatisfaction.

\section{Ethical considerations}

This study was conducted according to the principles of the Declaration of Helsinki. Information about the study was given to the patients in writing, together with the posted questionnaire. The patient information emphasized that their choice to participate or not was voluntary and that their decision would not influence their future treatment. The health professionals gave their verbal consent to participate in the study. Information about the study was given in writing as well as verbally. Both study groups were informed that presentation of the material would be anonymous so that statements could not be traced to any particular patient or team member. There was no contact between the respondents and researchers collecting the study data. The pros and cons of performing a telephone-based dropout analysis for nonresponders were thoroughly considered from an ethical point of view. The study was approved by the research ethics committee at Linköping, Sweden (M 138-05).

\section{Results}

\section{Patients' perspective}

The data from the patients revealed three categories: "the intervention is helpful for most and crucial for some"; "being listened to, respected, and validated", and "gives important coping strategies".

\section{Intervention is helpful for most and crucial for some} Nineteen of the patients described the intervention as helpful. Five of these patients described the intervention as crucial to their future: "I don't believe I would have survived if I hadn't been helped, as both my body and soul were aching all the time"; "Now I can cope with the pain when it shows up and I know that it eventually will diminish. I have also got a bite-splint that I think I should have had years ago." However, it is apparent that the patients in their narratives did not always discriminate between evaluation by the entire consultant team and efforts made by individual team members during follow-up, for example, support from the stress therapist.

"I can cope with the stress better; I don't get so scared when the vertigo comes. The support from the stress therapist makes me dare to plan for social activities even though I never know if the vertigo will show up." Two patients felt that they had been helped, but by events other than meetings with the consultant team. Two patients considered that they had not been helped at all.

\section{Being listened to, respected, and validated}

The patients reported that the most helpful component of the intervention was the respect, understanding, and validation they received from the consultant team. "The most helpful thing was to get to (talk about) your problems and be taken seriously." Some of the patients felt that it was the first time in a long history of suffering and health care contacts that they really felt respected, understood, and validated. "To meet the consultant team was like a revelation to me. At last someone who really saw you as whole human being, who took time, showed presence and interest."

\section{Provides important coping strategies}

For this category, three subcategories were identified, ie, "still have symptoms but can cope with them better", "better at handling stress", and "less worried and afraid when symptoms appear'. Most patients still had symptoms, with only a few reporting that their symptoms had remitted. About two thirds of the patients experienced symptom relief and one third reported that their symptoms were unchanged from before they met the consultant team. Most of the patients reported that they now had better coping strategies and could accept their symptoms better as a result of the intervention. The patients perceived pain differently, in that it was not quite so frightening once they had a model to understand it (ie, the psycho-neuro-endocrine-immune-model taught by the consultant team). "I got insight and understanding around how my body and soul reacts when I get stressed up and get into situations I believe I can't influence" and "They were asking necessary questions and made me see that change was needed".

\section{Health professionals' perspective}

Data from members of the consultant team revealed six categories: "the intervention helps most of the patients"; "to listen and respect the patients' experiences is crucial for the result"; "to balance earlier negative health care experiences"; "the multidisciplinary approach helps see the patient 
as a whole human being"; "the multidisciplinary approach brings security and job satisfaction"; and "giving hope but not false expectations is a hard balance".

\section{Intervention helps most patients}

The team members agreed that their work as part of the intervention team was helpful to most of the patients. In particular, they pointed out that they were trying to see the whole person and their life situation, not merely the symptoms. "You start with the person instead of the symptoms" and "It's about the whole life actually, much more than it's about teeth". The fact that patients were well prepared before they came to the session with the team was described as facilitating movement directly to the core of the problem.

\section{Listening to and respecting the patient's experience is crucial}

The team members reported being convinced that their encounter with the patients played a major role in the intervention. Listening, being respectful, and validating were described as components crucial to the result. "We treat the patients more like our guest of honor than a patient" and "... and I feel that we act more like interested fellow human beings than health care personnel, and I think that the patients sense that and it's making them feel secure".

\section{Balancing earlier negative health care experiences}

When the patient came to the session with the team, they often had a long history of negative experiences with health care, ie, not being listened to, not being understood, and not being helped. The team members described using the team session as an opportunity to break the cycle of negative experiences, mostly by just asking patients about themselves and listening to them ("... we almost always talk about experiences of insulting treatment from health care first ...").

\section{Multidisciplinary approach helps see the patient as a whole human being}

The consultant team described it as helpful for the patients to meet with and be seen by people representing different occupational groups within health care at the same time. The team comprised several different professions, and included both women and men of different age groups. This heterogeneous assembly was identified as a factor for success, for understanding the patients, and for minimizing the risk that something of relevance to a patient's problems would be overlooked. "Not just that we represent different occupations, but we are also different in age and represent different genders and experiences ... and we are actually using that." They also described how they took on different roles in the conversation, reflecting their different personalities. The psychologist, for example, often took the role of "the least informed" and asked clarifying questions of the rest of the team. "When X starts to look surprised and grimace, then we know that there is a risk that the patient has lost us and does not understand what we are talking about."

\section{Multidisciplinary approach brings security and job satisfaction}

The team reported that it was important to be able to make active choices about working in the team and that they were not selected to participate by someone else, such as a manager. Knowing this brought a profound sense of security to the team as well as job satisfaction. "We are absolutely not ordered here; on the contrary it feels like a silver lining of our work to be here."

There was also a sense of security in knowing that they were representing a wide range of specialties and that everybody was competent in their area. "When I'm all alone with a patient I'm like more vulnerable, I'm more afraid to do something, say something, or do something wrong." The team members were convinced that this feeling of security passes on to the patient, making it easier for the patient to feel comfortable talking about difficult issues before a group of people.

\section{Giving hope but not false expectations}

is a hard balance

The team perceived the intervention as giving patients some hope for the future. However, this could involve false expectations in some cases, because the action plan drawn up during the session might not be followed accurately by other people immediately involved with the patient. "We send our protocols out to the other actors and we contact them, even so it happens that it will not become like we planned and that's very frustrating for the patient and for us as well." This frustration arises because the team has tried to work on earlier negative health care experiences but ends up becoming one of these experiences.

\section{Discussion}

The aim of the present study was to describe patients' and health professionals' experiences of a multidisciplinary stress-focused clinical evaluation with prolonged engagement as an intervention for patients with long-term orofacial pain. A vast majority of the patients described the intervention 
as helpful. Some even reported that the meeting with the orofacial pain consultant team was crucial to their future. Most patients said they were still suffering from pain, with only a few reporting that their pain had fully remitted. However, they reported that, because of the intervention, they had developed more constructive coping strategies and were better able to accept their symptoms. They also described their perception of orofacial pain as being different, in that it was not so frightening when they had a model with which to understand it.

The concordance between the patients' and health professionals' experiences of the intervention is striking. In their descriptions, the health professionals and patients underscored the same "effective components", ie, understanding, respect, and validation. These aspects of a good patient-health professional relationship could be the result of the personality of the individual health professional and the beliefs they hold. However, in their narratives, the health professionals rejected this hypothesis by stressing that their way of viewing and encountering the patients lay within the model of the intervention. Given the importance of health professionals having knowledge relevant to their patients' problems, a growing body of research shows that respect, understanding, and validation are key to creating an alliance with patients, which in turn seems to be the foundation of being helpful. ${ }^{17-19}$ A qualitative study by Quirk et $\mathrm{al}^{20}$ identified 13 behaviors on the part of physicians that could be linked to the concept of being caring, as opposed to being uncaring. They found that this set of behaviors, representing a caring attitude, could be arranged in four categories, ie, being respectful, being empathetic, communicating effectively, and arranging to meet health care needs. However, they concluded that the most important element of caring might not be this set of behaviors per se, but rather an underlying philosophy of trying to see things from the patient's perspective, which corresponds well with the results of our present study.

The patent evaluation in the present study was also experienced as interventional. The capacity to intervene is probably reinforced by prolonged engagement. In the patients' narratives, when it came to descriptions about how the intervention had affected their well-being, patients seldom made any distinction between the session with the consultant team and follow-up with the stress therapist. The follow-up phase of the intervention also fitted well with the category of "arrange to meet health care needs" in an earlier study of caring attitudes. ${ }^{20}$

There is a long tradition in psychiatry and psychotherapy of regarding patients" narratives as a "source of healing".
In our experience, this is seldom the case in somatic care. Many health care researchers with a professional background in somatic care are astonished initially at the interventional power their interviews seem to have when they are performing a qualitative study addressing a patient group. One of them is the Swedish physiotherapist Maria Afrell who, whilst exploring patients' experiences of "living with a body in pain", found that her method of asking questions had interventional power. $^{21,22}$ In our study, the patients' descriptions indicate that the narrative approach taken by the consultant team might have been key to the success of the intervention. We do not know if writing about the experiences of health care had any additional effect in this study.

\section{Methodologic issues}

Qualitative research is concerned with the relationship between humans and their environment in all its complexity. It is based on the premise that, when trying to understand human beings, it is to the researcher's advantage to be able to describe experiences as they are narrated by the people themselves without being able to generalize the results to the population. ${ }^{23}$ The distinctive feature and strength of qualitative research is that it can provide a detailed description of intersubjective experiences and identify patterns from which hypotheses can emerge. ${ }^{24}$ Many of the strategies used to enhance trustworthiness in qualitative research focus on different perspectives (data sources, methods, investigators, or theories) to address the same topic. ${ }^{23,25}$ Different perspectives regarding data sources and methods of data collection were used in the present study. To enhance trustworthiness further, authors SE and KIP independently analyzed the material.

This study has some potential weaknesses and limitations that need to be addressed, in particular its retrospective nature, which is widely regarded as a major drawback in quantitative research. If registry or some other time-consistent reliable data source is not available, there is a considerable risk that subjective recall by patients could bias the results of a study. Because we tend to remember important details and forget those that are less important, so-called memory bias is not only a minor problem in qualitative research. The researcher is asking openly about the participants' personal experiences and not just about the details or specifics of interest to the researcher. We believe that there is potential for using qualitative research for evaluative purposes in clinical health research, but that this is often overlooked.

Dropout is a source of bias in many studies. When the aim of a study has an evaluative component, dropout is a 
major source of selection bias. Therefore, we performed a dropout analysis as part of the present study. Patients who did not respond to either the original invitation letter or the reminder were followed up by telephone to find out if their experiences differed in any major way from those of the responders. Only one of the five nonresponders indicated dissatisfaction with the intervention, which was considered to be the most important source of selection bias.

\section{Conclusion}

Most of the patients in this study described the intervention as helpful. Some reported that meeting with the consultant team was crucial. Most patients were still suffering from pain, with only a few reporting that their pain had fully remitted. However, because of the intervention, the patients considered that they had been provided with more constructive coping strategies and could accept their symptoms better. They also described their perception of pain as being different, and that the pain became less frightening when they had a model with which to understand it.

\section{Acknowledgments}

The authors are grateful to all the study respondents for kindly allowing us to take part in their stories. Financial support for this study was provided by the Division of Dental Care, Kalmar County Council, Sweden.

\section{Disclosure}

The authors report no conflicts of interest in this work.

\section{References}

1. Locker D, Slade G. Association of symptoms and signs of TM disorders in an adult population. Community Dent Oral Epidemiol. 1989;17: $150-153$.

2. Vickers ER, Boocock H. Chronic orofacial pain is associated with psychological morbidity and negative personality changes: comparison to the general population. Aust Dent J. 2005;50:21-30.

3. Lee LT, Yeung RW, Wong C, McMillan AS. Diagnostic sub-types, psychological distress and psychosocial dysfunction in southern Chinese people with temporomandibular disorders. J Oral Rehabil. 2008;35: 184-190.

4. Macfarlane TV, Blinkhorn AS, Davies RM, Kincey J, Worthington HV. Oro-facial pain in the community: prevalence and associated impact. Community Dent Oral Epidemiol. 2002;30:52-60.
5. Macfarlane TV, Blinkhorn AS, Davies RM, Kincey J, Worthington HV. Predictors of outcome for orofacial pain in the general population: a four-year follow-up study. J Dent Res. 2004;83:712-717.

6. Stohler CS. Chronic orofacial pain: is the puzzle unraveling? J Dent Educ. 2001;65:1383-1392.

7. Korszun A. Facial pain, depression and stress-connections and directions. J Oral Pathol Med. 2002;31:615-619.

8. Madland G, Feinmann C. Chronic facial pain: a multidisciplinary problem. J Neurol Neurosurg Psychiatry. 2001;71:716-719.

9. Dworkin SF. Behavioral and educational modalities. Oral Surg Oral Med Oral Pathol Oral Radiol Endod. 1997;83:128-133.

10. Turner JA, Jensen MP, Romano JM. Do beliefs, coping, and catastrophizing independently predict functioning in patients with chronic pain? Pain. 2000;85:115-125.

11. Turner JA, Mancl L, Aaron LA. Brief cognitive-behavioral therapy for temporomandibular disorder pain: effects on daily life, outcomes and process measures. $J$ Dent Res. 2005;86:58-63.

12. Lutgendorf SK, Costanzo SE. Psychoneuroimmunology and health psychology: an integrative model. Brain Behav Immun. 2003;17: $225-232$.

13. Goodkin K, Visser A. Psychoneuroimmunology in Stress, Mental Disorders and Health. Washington, DC: American Psychiatric Press; 2005.

14. Nyström C, Nyström O. [Self-assessed stress verifies autonomous dysfunction.] Skattad stress verifierar autonom dysfunktion. Läkartidningen. 1996;28-29:2583-2584. Swedish.

15. Burnard P. A method of analysing interview transcripts in qualitative research. Nurse Educ Today. 1991;11:461-466.

16. Cavanagh S. Content analysis; concepts, methods and application. Nurse Res. 1991;4:5-16.

17. Perseius KI, Öjehagen A, Ekdahl S, Åsberg M, Samuelsson M. Treatment of suicidal and deliberate self-harming patients with borderline personality disorder using dialectical behavioral therapy: the patients' and the therapists' perceptions. Arch Psychiatr Nurs. 2003;17: 218-227.

18. Perseius KI, Ekdahl S, Åsberg M, Samuelsson M. To tame a volcano: patients with borderline personality disorder and their perceptions of suffering. Arch Psychiatr Nurs. 2005;19:160-168.

19. Widar M, Ek AC, Ahlström G. Caring and uncaring experiences as narrated by persons with long-term pain after a stroke. Scand J Caring Sci. 2007;21:41-47.

20. Quirk M, Mazor K, Haley HL, et al. How patients perceive a doctor's caring attitude. Patient Educ Couns. 2008;72:359-366.

21. Afrell M, Biguet $\mathrm{G}$, Rudebeck CE. Living with a body in pain - between acceptance and denial. Scand J Caring Sci. 2007;21:291-296.

22. Afrell M, Rudebeck CE. 'We got the whole story all at once': physiotherapists' use of key questions when meeting patients with long-standing pain. Scand J Caring Sci. 2010;24:281-289.

23. Polit DF, Beck CT. Nursing Research, Principals and Methods, 8th ed. Philadelphia, PA: JB Lippincott Company; 2008.

24. Patton MQ. Qualitative Evaluation and Research Methods, 2nd ed. Newbury Park, CA: Sage Publications; 1991.

25. Sandelowski M. Rigor or rigor mortis: the problem of rigor in qualitative research revisited. Adv Nurs Sci. 1993;16:2-8.
Journal of Multidisciplinary Healthcare

\section{Publish your work in this journal}

The Journal of Multidisciplinary Healthcare is an international, peerreviewed open-access journal that aims to represent and publish research in healthcare areas delivered by practitioners of different disciplines. This includes studies and reviews conducted by multidisciplinary teams as well as research which evaluates the results or conduct of such teams or

\section{Dovepress}

healthcare processes in general. The journal covers a wide range of areas and welcomes submission from practitioners at all levels, from all over the world. The manuscript management system is completely online and includes a very quick and fair peer-review system. Visit http://www.dovepress.com/testimonials.php to read real quotes from published authors. 